\title{
L'inquilinisme chez les bourdons
}

\section{Par Patrick LHOMME *}

\begin{abstract}
In social insects, severe brood care costs have favoured the evolution of cheaters that exploit workers services of conspecifics or heterospecifics. In Bumblebees, a lot of species use hosts facultatively as an alternative to care for their own brood, while Psithyrus species have lost their worker caste and are completely dependant on hosts to produce their sexuals. Here is an overview of the adaptations and strategies used by bumblebees inquilines to successfully enter and exploit host social systems.
\end{abstract}

Résumé. Chez les insectes sociaux, le coût important des soins donnés aux couvains a favorisé, au cours de l'évolution, l'apparition d'espèces tricheuses capable d'exploiter la force de travail des colonies pour faire élever leur progéniture. Chez les bourdons, de nombreuses espèces usurpent des colonies de manière facultative. Toutefois les espèces du sous-genre Psithyrus ne produisent pas de caste ouvrière et sont totalement dépendantes de leurs hôtes pour la survie de leurs sexués. L'auteur présente ici une revue des différentes adaptations et stratégies utilisées par ces bourdons inquilines pour pénétrer et exploiter le système social de leurs hôtes.

Mots-clés. Inquilinisme, bourdons, Psithyrus, parasites sociaux.

\section{Introduction}

Les bourdons (Hymenoptera : Apinae : Bombini) sont des insectes eusociaux primitifs, avec un cycle de vie annuel. Au début du printemps, les reines émergent de leur hibernation et se mettent à la recherche d'un site de nidification pour fonder leur colonie. On peut voir assez régulièrement, chez les reines tardives, des comportements d'usurpation d'une colonie déjà existante. L'intruse supplante hiérarchiquement ou même tue la reine de la colonie hôte pour en prendre le contrôle. Ces individus exploitent ainsi non seulement les ressources alimentaires de leur hôte mais également la force de travail des ouvrières qui prendront soin de leur descendance. Ce type de comportement est particulièrement fréquent dans les milieux ou les sites de nidification sont en nombre restreints comparés au nombre de futures reines (Sladen 1912). Voveikov (1953) et Bohart (1970) ont même observés plusieurs cas de multiples successions de reines au sein d'un même nid. Ces cas d'inquilinisme facultatif s'observent aussi bien au sein de la même espèce qu'entre espèces différentes (Voveikov 1953; Alford 1975).

Certaines espèces ont même perdu toute capacité de fonder une colonie autonome. Elles sont devenues des inquilines obligatoires, on parle aussi de parasites sociaux. Le parasitisme social est apparu au moins à trois reprises de façon indépendante au cours de l'évolution des bourdons : (1) chez les Psithyrus, groupe constitué exclusivement d'espèces inquilines; (2) chez les Thoracobombus, avec B. inexspectatus (Yarrow 1970); (3) chez les Alpinobombus, avec B. hyperboreus (Richards K.W. 1973).

* Université de Mons-Hainaut, Place du parc 20, B-7000 Mons,

Belgique. E-mail : patrick.lhomme@umh.ac.be
L'explication généralement proposée sur l'origine de ce type de relations inquilines repose largement sur la règle d'Emery (1909). Cette règle explique l'apparition des parasites sociaux par un phénomène de spéciation sympatrique à partir d'une population d'un hôte social. Cette spéciation «parasitaire» expliquerait alors la coévolution entre l'hôte et son parasite (Schmid-Hempel 1998; Lowe et al. 2002). Ce principe se confirme chez de nombreux hyménoptères sociaux, surtout chez les fourmis (Lenoir et al. 2001), mais également chez certains bourdons. Les relations inquilines entre, par exemple, $B$. hyperboreus et $B$. polaris respectent étroitement cette règle. En revanche les Psithyrus la contredisent, puisqu'ils constituent un clade monophylétique au sein du genre Bombus (Cameron et al. 2007).

Se pose alors la question de la reconnaissance et de l'acceptation des Psithyrus par des hôtes éloignés d'un point de vue phylogénétique? D'autant que les Psithyrus sont dépourvues de corbeilles à pollen et ne produisent pas de caste ouvrière. Ils sont de ce fait totalement dépendants de leurs hôtes pour la survie de leur progéniture.

\section{Repérage du nid de l'hôte}

Les femelles Psithyrus émergent de leur hibernation un peu plus tard que leurs hôtes. Elles passent d'abord la plupart de leur temps à butiner, se nourrissant de nectar et de pollen. Puis, une fois leurs ovaires développés, elles se mettent à la recherche des nids de leurs hôtes (Alford 1975). Une femelle inquiline distingue une colonie hôte à partir de l'odeur des individus qui la compose (Fisher 1983a, 1985). Cederberg (1979, 1983) et Van Honk et al. (1981) ont montré que B. rupestris (Figure 1) et B. vestalis (Figure 2) trouvent l'entrée du nid en suivant les traces odorantes 


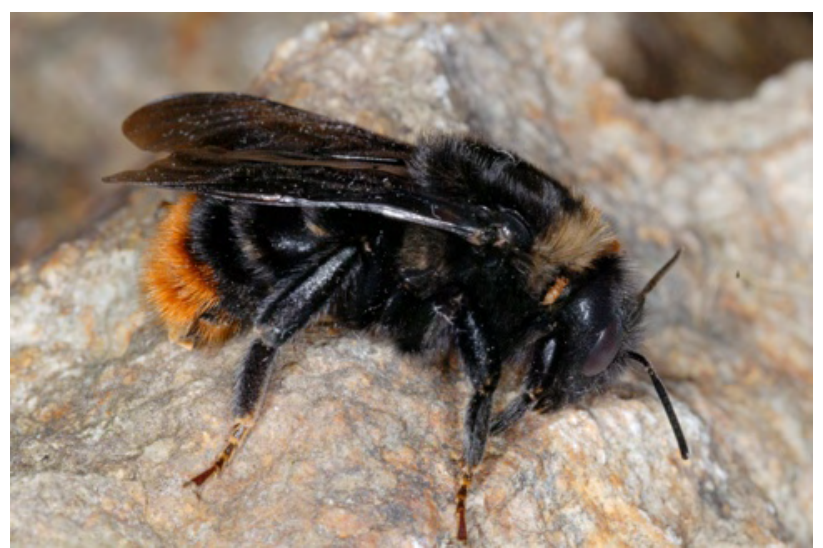

Figure 1. Femelle de Bombus rupestris armeniacus (Photo P Rasmont)

laissées par les ouvrières. Ces indices chimiques sont produits par la glande de Dufour au niveau du dernier tergite abdominal et sont reconnus de manière spécifique par les Psithyrus (Fisher et al. 1993). En règle générale, une espèce de Psithyrus ne parasite qu'une seule espèce, ou un petit groupe d'espèces proches appartenant au même sous-genre (Tableau 1).

\section{Pénétration dans la colonie}

Une fois le nid repéré, le Psithyrus évalue dans un premier temps la taille de la colonie hôte (Sramkova \& Ayasse 2009). Si celle-ci est trop grande, l'intruse serait rapidement éliminée par les ouvrières hôtes. Si la colonie est trop petite, il n'y aura pas assez d'ouvrières pour s'occuper des futures larves. Après avoir choisi la bonne colonie (10 à 15 individus), le Psithyrus doit ensuite être capable de détourner les signaux de reconnaissance de son hôte pour se faire accepter dans la colonie. Ce problème se retrouve à deux niveaux du cycle de vie des bourdons, (i) pour la reine qui doit envahir le nid de l'hôte et se faire accepter, mais aussi (ii) pour les jeunes sexués qui doivent vivre dans le nid jusqu'à leur émancipation.

Chez les bourdons, comme chez la plupart des hyménoptères sociaux, ce système de reconnaissance est basé sur la détection de composés chimiques lipidiques (hydrocarbures) recouvrant la cuticule (Singer 1998; Lenoir et al. 1999). Les individus de la colonie comparent les signaux perçus à un modèle de référence (ou «template») et attaquent tout individu rencontré si celui-ci dévie trop de ce standard. Ce template est le résultat de l'apprentissage d'un mélange d'odeurs partagées par tous les membres de la colonie et résultant d'un transfert réciproque de signaux chimiques entre les congénères (Dronnet et al. 2005). Le Psithyrus doit donc pouvoir contourner cette barrière chimique pour s'immiscer à l'intérieur du fonctionnement social de son hôte.

L'accueil qu'une femelle inquiline reçoit lorsqu'elle pénètre dans un nid hôte dépend de son propre comportement mais surtout de la taille de la colonie usurpée (Pouvreau 1973). C'est en général après la production de la première génération d'ouvrières que les bourdons inquilines usurpent une colonie. Les

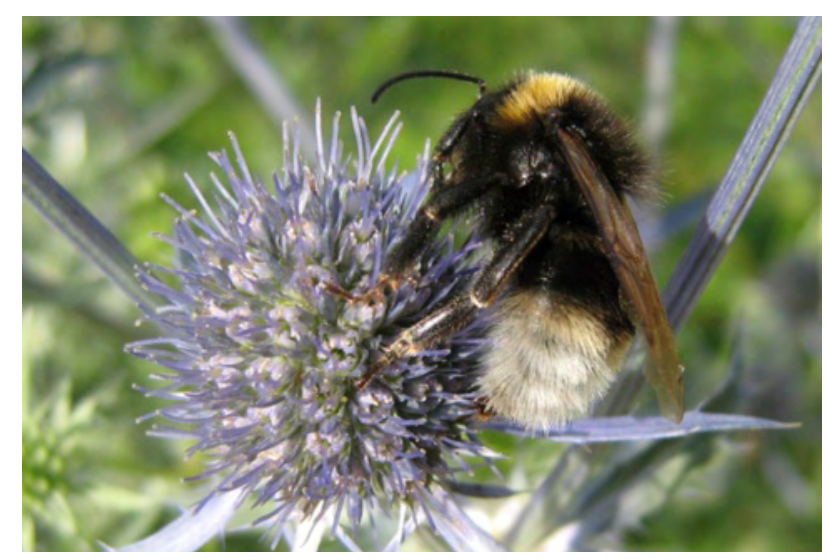

Figure 2. Mâle de Bombus vestalis (Photo P Rasmont)

premières ouvrières, qui n'ont pas encore de couvain à protéger, sont moins agressives envers les envahisseurs et sont également peu nombreuses, ce qui facilite l'usurpation des colonies (Alford 1975).

Généralement, une fois dans la colonie, les Psithyrus évitent tout contact avec leurs hôtes. Ils se dissimulent sous les matériaux du nid ou du couvain pendant quelque temps. Les Psithyrus s'imprègnent ainsi de l'odeur de la colonie, ce qui a pour effet de supprimer toute hostilité de la part des ouvrières (Fischer 1988; Küpper \& Schwammberger 1995). Ces inquilines emploient en fait une stratégie discrète : ils ne sont pas agressifs et ne possèdent aucune signature chimique sur leur cuticule, ce qui leur permet de ne pas êtres discriminés comme étrangers par leurs hôtes (Dronnet et al. 2005). Cette quasi-absence d'hydrocarbures cuticulaires leur permet également d'adopter rapidement l'odeur de la colonie. Certains Psithyrus, comme par exemple Bombus norvegicus (Figure 3), se protègent des attaques des ouvrières hôtes en sécrétant des allomones (substances de défense) produites par leur glande de Dufour (Zimma et al. 2003).

Dans d'autres cas, certaines femelles inquilines manifestent des comportements agressifs dès leur intrusion dans le nid, allant parfois jusqu'à la mise à mort de certains individus, notamment de la reine (Fisher 1984, 1988). Ces Psithyrus adoptent aussi des comportements de domination permettant d'inhiber l'agressivité des ouvrières : ils étreignent chaque ouvrière une à une en agitant leur dard («mauling behavior»). Ce comportement a été observé chez plusieurs espèces de Psithyrus ayant un mode d'usurpation agressif, notamment chez B. vestalis (Van Honk et al. 1981), B. bohemicus (Figure 4) (Fisher 1988) et les espèces américaines B. ashtoni (Fisher 1983b, 1987a) et B. citrinus (Fisher 1984). Les Psithyrus qui ont un mode d'usurpation plus pacifique comme $B$. campestris (figure 5 ) et $B$. sylvestris (Figure 6) ne présentent pas de tel comportement (Fisher 1988; Küpper \& Schwammberger 1995). Enfin plusieurs observations montrent que certains inquilines sont capable de vivre en apparente harmonie avec la reine hôte. Ce type de cohabitation à notamment été décrit chez B. campestris et B. sylvestris (Hoffer 1882, 1889; Alford 1975; Küpper \& Schwammberger 1995). 

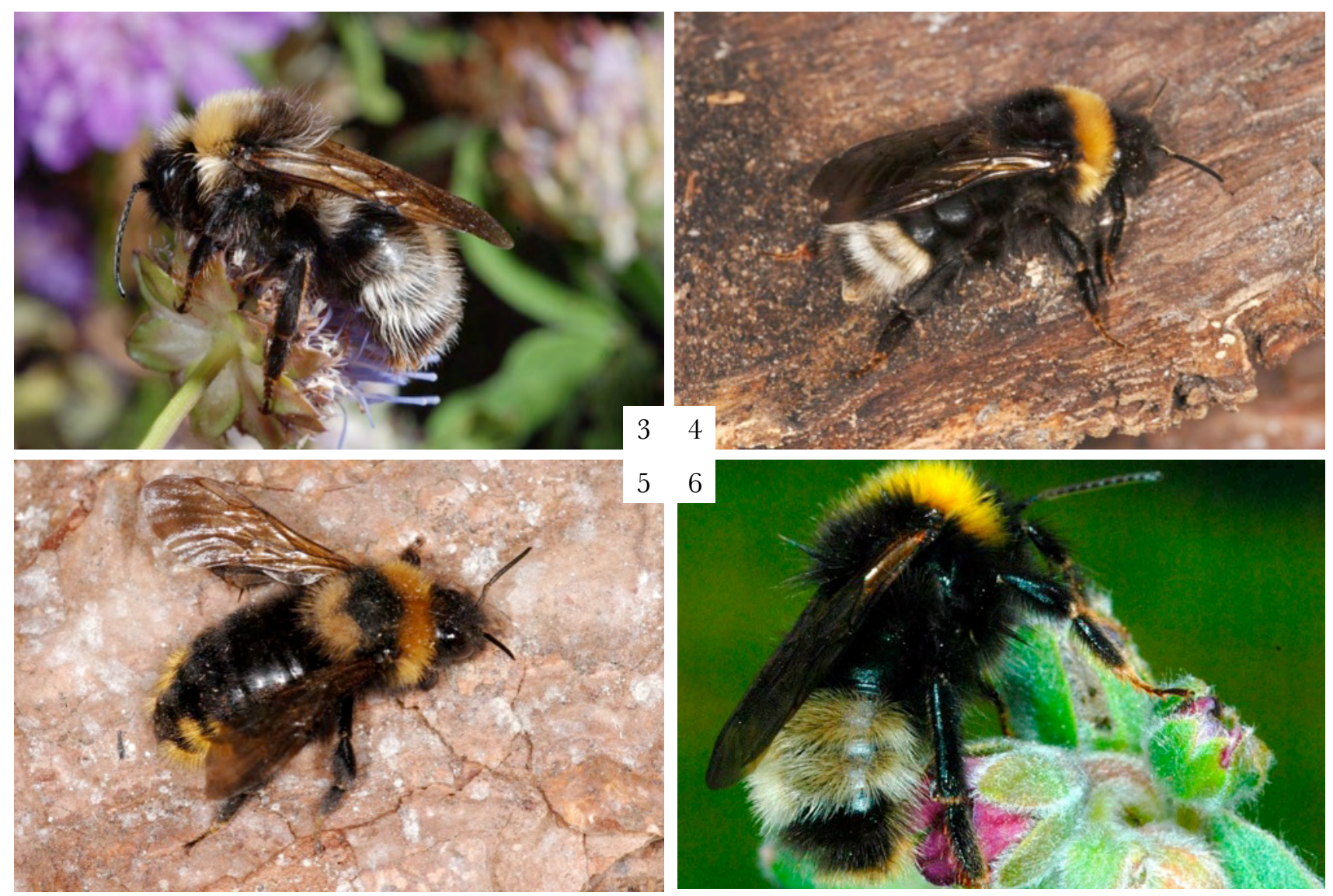

Figures 3 à 6. Quelques bourdons inquilines. 3. Mâle de Bombus norvegicus (Photo JC Schou); 4. Femelle de Bombus bohemicus (Photo P Rasmont); 5. Femelle de Bombus campestris (Photo P Rasmont); 6. Mâle de Bombus sylvestris (Photo A Pauly)

Le degré d'agressivité d'un inquiline semble dépendre de sa capacité à imiter les signaux chimiques des hôtes ou à produire les allomones spécifiques.

Dans tous les cas, il est assez rare que les femelles de Psithyrus soient tuées par les membres d'une colonie de bourdons. Ces véritables «machines de guerre» possèdent des caractères anatomiques leur permettant de résister aux attaques : cuticule plus épaisse, membranes intersegmentaires plus résistantes (surtout dans les régions vulnérables comme le cou ou le propodeum), mandibules plus acérées, dard plus puissant.

\section{Contrôle parental}

Une fois la reine remplacée, l'intruse détruit les oeufs et les larves de son hôte (Fisher 1987a) et se nourrit des provisions du nid. Dans les colonies ou les deux reines cohabitent, seuls les œufs de la reine hôte pondus après les premiers œufs du Psithyrus sont détruits par l'inquiline. La nouvelle reine inhibe également le développement ovarien des ouvrières (Fisher 1984, 1987b; Vergara et al. 2003). Son action inhibitrice ne semble cependant pas aussi efficace que celle exercée par la reine hôte. Après quelques jours, la femelle Psithyrus dépose sa première ponte dans une cellule cireuse qu'elle édifie elle-même à partir des débris de cocons. Dans un premier temps, elle incube, nourrit et protège ses propres larves contre les ouvrières (Fisher 1987b, 1988; Küpper \& Schwammberger 1995). En fin de cycle, seules les ouvrières approvisionnent les larves et les adultes de Psithyrus en miel et pollen.

Aucune étude ne montre quelles stratégies les larves adoptent pour se faire accepter et même nourrir par les ouvrières. Une étude récente de Cervo et al. (2008) montre que les larves du parasite social Polistes sulcifer (Hymenoptera : Vespidae) adoptent une stratégie de camouflage différente de celle de leur mère. Contrairement aux adultes, les larves sont obligées d'avoir un taux d'hydrocarbures important sur leur cuticule pour les prévenir de la dessiccation. A la différence de leur mère, dont la stratégie est de n'avoir aucune signature chimique («odorless hypothesis»), les larves naissent avec une identité chimique propre mais qui n'est pas reconnue par l'hôte («neutral odor hypothesis»). On pourrait imaginer qu'il en soit de même pour les larves de Psithyrus. L'hypothèse reste à tester.

Le nombre de futurs sexués produits par une femelle Psithyrus dépend de son potentiel de ponte ${ }^{1}$, mais aussi du nombre et de l'âge des ouvrières (Pouvreau 1973; Van Honk et al. 1981). Plus le Psithyrus usurpe une colonie tardivement, plus le nombre d'ouvrières pondeuses en fin de cycle est important et donc plus le contrôle parental est difficile. Après intrusion dans une colonie, les Psithyrus sont d'ailleurs capables de tuer sélectivement les ouvrières les plus âgées, qui risquent 
Tableau 1. Relations hôtes-inquilines entre les espèces de Bombus et de Psithyrus présentes en France. Les astérisques (*) indiquent des hôtes occasionnels.

\begin{tabular}{|c|c|c|}
\hline Psithyrus & Bombus hôtes & Principales références bibliographiques \\
\hline \multicolumn{3}{|l|}{ Allopsithyrus } \\
\hline \multirow[t]{3}{*}{ B. barbutellus } & B. (Megabombus) hortorum & Sladen 1912; Ball 1914; Müller 1936; Cumber 1949; Postner 1952; Løken 1984 \\
\hline & B. (Megabombus) ruderatus* & Westrich 1989; Peeters et al. 1999 \\
\hline & B. (Pyrobombus) hypnorum* & Pouvreau 1973; Løken 1984 \\
\hline \multirow[t]{2}{*}{ B. maxillosus } & B. (Megabombus) ruderatus & Reinig 1935; Pittioni \& Schmidt 1942; Ornosa 1984; Rasmont \& Adamski 1996 \\
\hline & B. (Megabombus) argillaceus* & Reinig 1935; Pittioni \& Schmidt 1942 \\
\hline \multicolumn{3}{|l|}{ Ashtonipsithyrus } \\
\hline B. bohemicus & B. (Bombus) lucorum & Sladen 1912; Reinig 1935; Cumber 1949; Pouvreau 1973; Løken 1984 \\
\hline B. perezi & B. (Bombus) terrestris xanthopus & Ferton 1901; Rasmont \& Adamski 1986 \\
\hline B. vestalis & B. (Bombus) terrestris & Sladen 1912; Reinig 1935; Pouvreau 1973; Van Konk et al. 1981; Løken 1984 \\
\hline \multicolumn{3}{|l|}{ Fernaldaepsithyrus } \\
\hline \multirow[t]{2}{*}{ B. flavidus } & B. (Pyrobombus) jonellus & Brinck \& Wingstrand 1951 \\
\hline & B. (Pyrobombus) monticola* & $\begin{array}{l}\text { Richards O.W. 1928; Pittioni 1942, 1943; Tkalcu 1969; Lundberg \& Svensson } \\
\text { 1977; Pekkarinen et al. } 1981\end{array}$ \\
\hline \multirow[t]{2}{*}{ B. norvegicus } & B. (Pyrobombus) hypnorum & Stoeckhert 1954; Röseler 1972; Cederberg 1976; Løken 1984 \\
\hline & B. (Pyrobombus) jonellus* & Brinck \& Wingstrand 1951 \\
\hline B. quadricolor & B. (Kallobombus) soroeensis & Hoffer 1889; Reinig 1935; Løken 1984 \\
\hline \multirow[t]{2}{*}{ B. sylvestris } & B. (Pyrobombus) pratorum & Hoffer 1889; Richards O.W. 1928; Pouvreau 1973; Alford 1975; Løken 1984 \\
\hline & B. (Pyrobombus) jonellus* & Richards O.W. 1928; Reinig 1935; Løken 1984 \\
\hline \multicolumn{3}{|l|}{ Metapsithyrus } \\
\hline B. campestris & B. (Thoracobombus) pascuorum & Hoffer 1889; Cumber 1949; Pouvreau 1973; Alford 1975; Løken 1984 \\
\hline & B. (Thoracobombus) humilis & Hoffer 1889; Løken 1984 \\
\hline & B. (Pyrobombus) pratorum & Pouvreau 1973; Løken 1984 \\
\hline & B. (Rhodobombus) pomorum & May 1937; Løken 1984 \\
\hline \multicolumn{3}{|l|}{ Psithyrus } \\
\hline \multirow[t]{4}{*}{ B. rupestris } & B. (Melanobombus) lapidarius & Hoffer 1889; Sladen 1912; Reinig 1935; Bols 1939; Pouvreau 1973; Alford 1975 \\
\hline & B. (Melanobombus) sichelii* & Bullman 1953; Móczár 1977 \\
\hline & B. (Thoracobombus) sylvarum* & Höppner 1901; May 1959 \\
\hline & B. (Thoracobombus) pascuorum* & Haeseler 1970 \\
\hline
\end{tabular}

d'entrer en compétition avec la reine pour la reproduction (Sramkova \& Ayasse 2009). Ils reconnaissent les ouvrières dominantes grâce aux signaux de fertilité qu'elles sécrètent (Sramkova et al. 2008). Ils maximisent ainsi leur succès reproducteur.

Une fois le développement larvaire terminé, les mâles sortent les premiers du nid au bout d'environ une semaine, suivis par les femelles quelques jours plus tard. Après leur envol, les jeunes femelles s'accouplent dans des territoires marqués par les mâles (Svensson 1980), puis recherchent un lieu d'hibernation.

\section{Origine du parasitisme social chez les bourdons}

L'évolution vers la vie inquiline ne paraît pas très étonnante au regard de la biologie des bourdons. On passe de comportements occasionnels imposés par les conditions climatiques (bivouac) à l'hébergement forcé, voire à l'élimination de la reine hôte. Cette grande diversité des comportements d'usurpation permet de retracer un chemin évolutif vers l'apparition du parasitisme social :
- Premier stade (les inquilines facultatifs) : l'usurpatrice tue la reine hôte. Il n'y a pas d'interaction initiale entre l'intruse et les ouvrières, seules les larves sont présentes au moment de l'invasion. L'usurpatrice récolte du pollen et nourrit ses larves. Elle produit des ouvrières et des sexués;

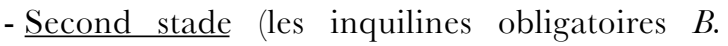
inexspectatus et $B$. hyperboreus) : l'usurpatrice ne produit pas d'ouvrières, mais semble toujours capable de le faire dans des milieux favorables. Elle possède des corbeilles à pollen, mais qui sont non fonctionnelles. L'intruse ne récolte pas de pollen pour ses larves;

- Troisième stade (les parasites sociaux du sousgenre Psithyrus) : l'intruse détruit le couvain et élimine la reine hôte. Elle ne produit pas d'ouvrières, ses glandes cirières sont atrophiées et les corbeilles à pollen sont absentes. Le soin donné au couvain est rudimentaire ou inexistant. 
Comment expliquer cette évolution vers le parasitisme social chez les bourdons ? On peut imaginer qu'au cours de l'évolution, les coûts important des soins donnés aux couvains (incubation, nourrissage, protection des larves) aient engendré l'apparition de stratégies «tricheuses» chez certaines espèces de bourdons, facilitant leur capacité d'usurpation de colonies déjà existantes. La sélection a pu alors conduire à la fixation de ces espèces tricheuses dans les populations et mener à la dérive de l'interaction vers du parasitisme obligatoire.

\section{Remerciements}

Je remercie Pierre Rasmont, Alain Pauly (Université de Mons, Belgique) et Jens Christian Schou (Hobro, Danemark) pour les photos qui illustrent cet article. Je remercie également Pierre Rasmont et Denis Michez (Université de Mons, Belgique) pour avoir accepté de relire et corriger cet article.

\section{Références bibliographiques}

Alford DV, 1975. Bumblebees. Davis-Poynter Ltd, London.

Ball FJ, 1914. Les Bourdons de la Belgique. Annales de la Société entomologique de Belgique 58: 77-108.

Bohart GE, 1970. The evolution of parasitism among bees. Utah state University, 30 pp.

Bolotov IN \& Kolosiva YS, 2006. Trends in the formation of biotopic complexes of bumblebees (Hymenoptera, Apidae: Bombini) in northern taiga karst landscapes of the western russian plain. Ekologiva 3: 173-183

Bols JH, 1939. Un remarquable terrain d'hivernation de Bombus et de Psithyrus près de Louvain, à Lubbeek, en Belgique. VIIth lnternational Congress of Entomology 1938: 1048-1060.

Brinck P \& Wingstrand KG, 1951. The mountain fauna of the Virihaure area in Swedish Lapland. Acta Universitatis Lundensis 45(2): 1-69.

Bullman 0, 1953. Psithyrus rupestris F. als Schmarotzer bei Bombus alticola KREICHB. Mittheilungen des Naturwissenschaft Vereins für Steiermark 83 : 193-194

Gameron SA, Hines HM, \& Williams PH, 2007. A comprehensive phylogeny of the bumble bees (Bombus). Biological Fournal of the Linnean Society 91: 161-188.

Cederberg B, 1976. Snylthumlan Psithyrus norvegicus sp. Schn., boparasit hos humlan Bombus hypnorum L. (Hym., Apidae). Entomologisk Tidsktift 97: 90-91.

Cederberg B, 1979. Odour guided host selection in Psithyrus (Hym., Apidae). Entomologisk Tidskrift 100: 128-129.

Cederberg B, 1983. The role of trail pheromones in host selection by Psithyrus rupestris (Hymenoptera, Apidae). Entomologica Fennica 49(1): 11-16

Cervo R, Dani FR, Cotoneschi G, Scal G, Lotti I, Strassmann JE, Queller DG \& Turillazzi S, 2008. Why are the larvae of the social parasite wasp Polistes sulcifer not removed from his host nest? Behavioral Ecology and Sociobiology 62: 1319-1331.

Cumber RA, 1949. Humble-bee parasites and commensales found within a thirty miles radius of London. Proceedings of the Royal entomological Society of London A 24(10-12): 119-127.

Dronnet S, Simon X, Verhaeghe JC, Rasmont P \& Errard C, 2005. Bumblebee inquinilism in Bombus (Fernaldaepsithyrus) sylvestris (Hymenoptera, Apidae): behavioural and chemical analyses of host-parasite interactions. Apidologie 36: 59-70.

Emery C, 1909. Über den Ursprung der dulotischen, parasitischen und myrmecophilen Ameisen. Biologishes Zentralblatt 29: 352-362.

Fisher RM, 1983a. Recognition of host nest odour by the bumble bee social parasite Psithyrus ashtoni (Hymenoptera: Apidae). Fournal of the New York Entomological Society 91: 503-507.

Fisher RM, 1983b. Inability of the social parasite Psithyrus ashtoni to suppress ovarian development in workers of Bombus affinis (Hymenoptera : Apidae). Fournal of the Kansas Entomological Society 56: 69-73.

Fisher RM, 1984. Dominance by a bumble bee social parasite (Psithyrus citrinus) over workers of its host (Bombus impatiens). Animal Behavior 32: 304-305.
Fisher RM, 1985. Evolution and host specificity: dichotomous invasion success of Psithyrus citrinus (Hymenoptera: Apidae), a bumblebee social parasite in colonies of its two hosts. Canadian fournal of Zoology 63: 977-981.

Fisher RM, 1987a. Queen-workeer conflict and social parasitism in bumble bees (Hymenoptera: Apidae). Animal Behavior 55: 1026-1036.

Fisher RM, 1987b. Temporal dynamics of facultative social parasitism in bumble bees. Animal Behavior 35: 1928-1636.

Fisher RM 1988. Observations on the behaviours of three European cuckoo bumble bee species (Psithyrus). Insectes Sociaux 35: 341-354.

Fisher RM, Greenwood DR \& Shaw GJ, 1993. Host recognition and the study of a chemical basis for attraction by cuckoo bumble bees (Hymenoptera: Apidae). Fournal of Chemical Ecology 19(4): 771-786.

Fisher RM \& Sampson BJ, 1992. Morphological specializations of the bumble bee social parasite Psithyrus ashtoni (CRESSON) (Hymenoptera : Apidae). Canadian Entomologist 124(1): 69-77.

Haeseler V, 1970. Zur wirtewhal des Psithyrus rupestris FAB. (Hymenoptera Apidae). Fauna Ökologie Mitteilungen III (9-10): 296-298.

Hoffer E, 1882. Biologische Beobachtungen an Hummeln und Schmarotzerhummeln. Mitteilungen Naturwissenschaftlicher Verein Steiermark 18: 69-92.

Hoffer E, 1889. Die Schmarotzerhummeln Steiermarks. Lebensgeschichte und Beschreibung derselben. Mitteilungen Naturwissenschaftlicher Verein Steiermark 25, 82-158.

Höppner H, 1901. Beiträge zur Bienenfauna der Lüneburger Heide. Fahresbericht Verhandlungen Naturkunden Unterweser 1900: 9-22.

Küpper G \& Schwammberger KH, 1995. Social parasitism in bumble bees (Hymenoptera, Apidae): observations of Psithyrus sylvestris in Bombus pratorum nests. Apidologie 26: 245-254.

Lenoir A, D'Ettorre P, Errard C \& Hefetz A, 2001. Chemical ecology and social parasitism in ants. Annual Review of Entomology 46: 573-599.

Løken A, 1984. Scandinavian species of the genus Psithyrus (Hymenoptera: Apidae). Entomologica Scandinavica 23: 1-45.

Lowe RM, Ward SA \& Crozier RH, 2002. The evolution of parasites from their hosts : intra- and inter-specific parasitism and Emery's rule. Proceedings of the Royal entomological Society of London B 269: 1301-1305.

Lundberg H, \& Svensson BG, 1977. Altitudinal distribution of Psithyrus Lep. in relation to that of their hosts Bombus LATR. in a Subalpine/Alpine area. VIIIth international Congress of IUSSI 1977: 305-306.

May J, 1937. Bionomie rodu Bombus LATR. a Psithyrus LEP. (1). Casopis Ceskoslovenské Spolecnosti Entomologické 34: 115-118.

May J, 1959. Cmelaci v. CSR jejich bionomie, chow a hos-podarsky vyznam. Praha. 170pp.

Móczár L, 1977. Das nest von Bombus alticola KRIECHBAUMER (Hymenoptera : Apidae). Acta Biologica Szegediensis 23 : 133-138

Müller M, 1936. Psithyrus barbutellus KIRBY und P. maxillosus KLUG. (Apid., Hym.). Mitteilungen der deutschen entomologishen Gesellschaft 6: 73-76.

Ornosa GC, 1984. La subfamilia Bombinae (Hym., Apidae) de la fauna espanola. Thèse, Universidad Complutense de Madrid, 333pp.

Peeters TMJ, Raemakers IP \& Smit J, 1999. Voorlopige atlas van de Nederlandse bijen (Apidae). European Invertebrate Survey Nederland, Leiden, 230 pp.

Pekkarinen A, Teräs I, Viramo J \& Paatela J, 1981. Distribution of bumblebees (Hymenoptera, Apidae: Bombus and Psithyrus) in eastern Fennoscandia. Notulae Entomologicae 61: 71-89.

Pittioni B, 1942. Die boreoalpinen Hummeln und Schmarotzerhummeln (Hymen., Apidae, Bombinae). I. Mitteilungen aus den Köninglichen Naturwissenschaftlichen Instituten in Sofia 15: 155-218.

Pittioni B, 1943. Die boreoalpinen Hummeln und $\mathrm{S}$ chmarotzerhummeln (Hymen., Apidae, Bombinae). II. Mitteilungen aus den Köninglichen Naturwissenschaftlichen Instituten in Sofia 16: 1-78.

Pittioni B \& Schmidt R, 1942. Die Bienen des südöstlichen Niederdonau. I: Apidae, Podaliriidae, Xylocopidae und Ceratinidae. Natur und Kultur Niederdonau 19: 1-69.

Postner M, 1952. Biologish-ökologishe untersuchen an Hummeln und ihre Nestern. Veröfentlichungen museum für Naturkunde 2: 45-86.

Pouvreau A, 1973. Les ennemis des bourdons. I.- Etude d'une zoocénose : le nid de bourdons. Apidologie 4(2): 103-148.

Rasmont P \& Admaski A, 1996. Les bourdons de la Corse (Hymenoptera, Apoidea, Bombinae). Notes fauniques de Gembloux 31: 3-87.

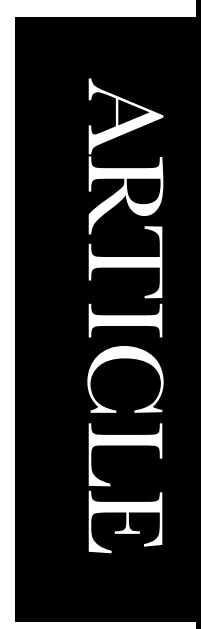


Reinig WF, 1935. On the Variation of Bombus lapidarius L. and its Cuckoo, Psithyrus rupestris FABR., with Notes on Mimetic Similarity. Fournal of Genetics 30(3): 321-356.

Richards KW, 1973. Biology of Bombus polaris CURTIS and B. hyperboreus SCHÖNHERR at Lake Hazen, Northwest Territories (Hymenoptera : Bombini). Quaestiones Entomologicae 9: 115-157.

Richards OW, 1928. A revision of the european bees allied to Psithyrus quadricolor LEPELETIER (Hymenoptera, Bombidae). Transactions of the Royal entomological Society of London 76: 345-365.

Röseler PF, 1972. Beobachtungen über die Schmarotzerhummeln Psithyrus norvegicus in einem nest der hummeln Bombus hypnorum (Hymenoptera: Apidae). Mittel Badischer Landesverein Naturkunde und Naturschultz 10: 579-581.

Schmid-Hempel P, 1998. Parasites in Social Insects. Princeton University Press, Princeton, New Jersey.

Singer TL, 1998. Roles of hydrocarbons in the recognition systems of insects. American Zoologist 38: 394-405

Sladen FWL, 1912. The Humble-Bee, its life-history and how to domesticate it, with descriptions of all the British species of Bombus and Psithyrus. MacMillian, London, 283 pp.

Sramkova A \& Ayasse M, 2009. Chemical ecology involved in the invasion success of the cuckoo bumblebee Psithyrus vestalis and in survival of workers of its host Bombus terrestris. Chemoecology (sous presse)

Sramkova A, Schulz C, Twele R, Francke W \& Ayasse M, 2008. Fertility signals in the bumblebee Bombus terrestris (Hymenoptera: Apidae). Naturwissenschaften 95(6): 515-522.

Stoeckhert FK, 1954. Fauna Apoideorum Germaniae. Abhandlungen der bayerischen Akademie der Wissenschaften 65: $1-87$

Svensson BG, 1980. Species-isolating mechanisms in male bumble bees (Hymenoptera, Apidae). Ph-D Thesis, Uppsala University, Uppsala, 42 pp.

Tkalcu B, 1969. Ergebnisse der Albanien-Expedition 1961 des deutschen Entomologischen Institutes. 78. Beitrag. Hymenoptera: Apidae IV (Bombinae). Beiträge zur Entomologie 19(7/8): 887-916.

Van Honk C, Röseler PF, Velthuis H \& Malotaux M, 1981. The conquest of a Bombus terrestris colony by a Psithyrus vestalis female. Apidologie 12: 57-68.

Vergara CH, Schroder S, Almanza MT \& Wittmann D, 2003. Suppression of ovarian development of Bombus terrestris workers by B. terrestris queens, Psithyrus vestalis and Psithyrus bohemicus females. Apidologie 34: 563-568.

Voveikov GS, 1953. Estestvennaya smena samok vo cem'ya Schmelej (Hym. Bomb.). Entomologzrheskoe Obozrenie 33: 174-181.

Westrich P, 1989. Die Wildbienen Baden-Württembergs. Spezieller Teil: Die Gattungen und Arten. Eugen Ulmer GmbH \& Co, Stuttgart, 972p.

Yarrow IHH, 1970. Is Bombus inexspectatus (TKALCU) a workerless parasite? Insectes Sociaux 17: 95-112.

Zimma BO, Ayasse M, Tengo J, Ibarra F, Schulz G \& Francke W, 2003. Do social parasitic bumblebees use chemical weapons? Fournal of Comparative Physiology 189: 769775 . 